\title{
Detecting Alcohol and Drug Use with the Touch of a Finger
}

\author{
Todd D. Pizitz ${ }^{1}$, Timothy A. Scheuber ${ }^{2}$, Court Wallner ${ }^{1}$, Kathleen Fernandez ${ }^{3}$ \\ ${ }^{1}$ Veracity Security Systems, LLC, San Diego, California, USA \\ ${ }^{2}$ Alliant International University, Clinical Psychology, San Diego, California, USA \\ ${ }^{3}$ Martse Training Centers, Chemical Dependency, San Diego, California, USA
}

Email address:

tpizitz@sbcglobal.net (T. D. Pizitz)

\section{To cite this article:}

Todd D. Pizitz, Timothy A. Scheuber, Court Wallner, Kathleen Fernandez. Detecting Alcohol and Drug Use with the Touch of a Finger. American Journal of Applied Psychology. Vol. 4, No. 4, 2015, pp. 90-94. doi: 10.11648/j.ajap.20150404.12

\begin{abstract}
The present study measured the accuracy and utility of the Veracity TouchScreener ${ }^{\mathrm{TM}}$ technology as a viable alternative to urine drug testing. 101 participants enrolled in court-ordered drug and alcohol treatment participated in the current study. All participants were administered 18 questions on a the Veracity TouchScreener ${ }^{\mathrm{TM}}$ and then participated in a visually monitored drug and alcohol urine test. The results revealed $92 \%$ accuracy at classifying alcohol and drug user's self-report of sobriety by using this new technology. Clinical implications for the treatment of alcohol and drug users are examined through the implementation this technology.
\end{abstract}

Keywords: Drug Use, Alcohol Abuse, Drug Detection, Technology

\section{Introduction}

The use of drugs and alcohol continues to have negative consequences to those afflicted with the disease of addiction and a widespread impact on society as a whole. In the general population, the lifetime prevalence of substance related disorders include estimates as high as $15 \%$ for alcohol dependence, $5 \%$ for cannabis dependence, $2 \%$ for cocaine, $1.5 \%$ for amphetamines, and approximately $2 \%$ for opioids (American Psychiatric Association, 2000). Interventions aimed at substance use cessation and relapse prevention include drug and alcohol treatment combined with some form of drug testing. Due to a plethora of motives - fear, shame, manipulation, and not wanting to be sober - people lie and are deceptive about their alcohol and drug consumption. With this in mind, substance abuse and honesty can be mutually exclusive. Therefore, professionals treating alcohol and drug-using individuals recognize the importance of not merely taking someone's word about his or her drug and/or alcohol intake. Rather, drug testing can help put an end to the games and guesswork that so often accompany treatment with drug and alcohol users. Of the available methods to measure substance consumption (urine, hair samples, blood, and oral swabs), urine appears to be the most commonly utilized method of drug detection. (Lum \& Mushlin, 2004). Most urinalysis tests offer confirmatory laboratory analyses for initial screenings that produce a positive result. The procedures used in this analysis are considered the gold standard as they provide a quantitative measure of how much of a substance or the corresponding metabolites were present in the test taker at the time of the screening (National Institute on Drug Abuse (NIDA), 1986).

The origins of urine drug testing began in the early 1970's with the military introducing urine specimen collection to Vietnam veterans. The military's 1971 Operation Golden Flow, aimed at detecting substance dependent veterans, was initiated to assist in reducing the use of heroin among military personnel. This anti-drug campaign, by use of urine drug testing, led to regular and random urine drug testing of military members that is still in effect today. Another event in American history that helped establish the roots of urine drug testing is when President Reagan issued an executive order in 1986 warning "the use of illegal drugs, on or off duty, by federal employees in certain positions...may pose a serious risk to national security" (MacDonald, 2013, P. 11). This order mandated all United States Agencies to implement urine drug testing. Following the mandate, urine drug testing ignited and became a widely accepted practice in many fields and disciplines.

Although urine drug testing with confirmatory laboratory analysis is the most widely used drug screening instrument this system is not without its limitations. Cutoff measurements, 
established by the Department of Health and Human Services (Office of the Federal Register, 2011), reference when a urine drug test result crosses a specified threshold thereby rendering the result positive for the presence of the measured substance. Detection times refer to the length of time a substance may be detected in the urine after it was last used. Since detection times vary with each substance (Verstraete, 2004), a urine drug test might not capture a positive result if the test is administered outside of the drug detection time window. In addition, certain substances may also cross-react with medications or other drugs leading to false-positive results (Lum \& Mushlin, 2004). Other limitations of urine drug testing are related to the known methods to adulterate and invalidate urine samples (Venkatratnam \& Lents, 2011). These limitations can cause treatment providers to assume clients are progressing favorably when they are not or make erroneous conclusions about drug use that has not actually occurred (Melanson, Baskin, Magnani, Kwong, Dizon, \& Wu, 2010). Other drawbacks to urine drug testing are related to the sample collection process, which can be intrusive and embarrassing, as most protocols require visual observation. Urine sample collection also requires significant time and labor in the process of collection and in maintaining a verifiable chain-of-custody. Utilizing other alternatives that do not have these limitations can prove useful to those who are supervising or treating individuals with alcohol or drug use behaviors and proclivities.

As an alternative to urine drug testing, Veracity Security Solutions, LLC devised touch screen technology that is capable of providing insight into individuals' reported drug and alcohol use by the touch of a single finger. The Veracity TouchScreener ${ }^{\mathrm{TM}}$ measures psychophysiological information on how a person emotionally reacts to a structured set of questions displayed on a specialized touch-screen computer by answering "Yes" or "No" questions regarding drug and alcohol consumption.

Initial validation research (Pizitz, McCullaugh, Sprague, Vaccaro, Blue, Mealing, \& Fernandez, 2014) demonstrated that computer-based, touch-screen technology is a viable alternative to urine drug testing. Pizitz et al. (2014) measured deception among 77 alcohol and drug users who were court-ordered for treatment by utilizing the Veracity TouchScreener ${ }^{\mathrm{TM}}$ and a standard visually monitored alcohol and drug urine test. The TouchScreener ${ }^{\mathrm{TM}}$ demonstrated an estimated $92 \%$ accuracy at classifying alcohol and drug user's self-report of sobriety (Pizitz et al., 2014).

The current study intended to replicate the findings from Pizitz et al. (2014) by demonstrating the utility of the TouchScreener ${ }^{\mathrm{TM}}$ in measuring the presence of potential drug and alcohol use among a sample of clients receiving court-ordered drug and alcohol treatment. For the purposes of this research, the authors examined the consistency between alcohol and drug users' self-reported claims of sobriety through their responses on the Veracity TouchScreener ${ }^{\mathrm{TM}}$ and the external criterion of a standard 8-panel alcohol and drug urine test.

\section{Methods}

\subsection{Participants}

The participants consisted of 101 male and female alcohol and drug users who were currently involved in two court-ordered sobriety maintenance programs. Inclusion criteria consisted of involvement in a court-ordered sobriety maintenance program, the ability to read English, and willingness to participate in a visually monitored alcohol and drug urine test. All participants were selected from two drug and alcohol sobriety maintenance programs in coastal Southern California. The demographic data related to age, gender, ethnicity, relationship status, and alcohol and drug urine test results were additionally gathered and are presented below, Table 1.

\subsection{Procedure}

Informed consent was obtained from the participants after a clear explanation of the study aim, procedure, and potential risks and benefits. At the onset of participation in the study, all participants signed an informed consent to participate in the research and completed a demographic questionnaire. Each participant agreed to the terms of the research and was aware that all identifying information would be confidential and used for research purposes. Participants answered 18 Yes or No questions on the TouchScreener ${ }^{\mathrm{TM}}$, which measured their self-reported sobriety (Appendix A). Subsequently, participants then provided a visually monitored urine specimen which was then sent to a laboratory for processing and the results were received via facsimile. To incentivize participation, each participant was provided a compensation of ten dollars $(\$ 10.00)$.

\subsection{Measures}

\subsubsection{Veracity TouchScreener ${ }^{\mathrm{TM}}$}

The Veracity TouchScreener ${ }^{\mathrm{TM}}$ is a computer touch screen that measures Significant Psychophysiological Responses (SPR) to a set of questions. Based on principles similar to polygraph examinations and voice stress analysis and involuntary muscle movements (Kelly, Murray, Barrios, Gorman, Ganis, and Keenan, 2008) the Veracity TouchScreener ${ }^{\mathrm{TM}}$ was developed to measure deception. This highly sensitive touch screen amplifies and measures small, but significant, variations in the person's touches as they answer the questions. Participants in the present study answered 18 Yes or No questions by touching their response to each item using one finger. The administration of the 18 questions via the Veracity TouchScreener ${ }^{\mathrm{TM}}$ involved an estimated 120 seconds to complete for each participant. Following the completion of answering the questions, each participant's responses were analyzed using a proprietary algorithm designed to assess psychophysiological indicators for each test item. The analysis revealed whether participants answered Yes or No to the items as well as assessing the number of psychophysiological indicators for relevant questions on the TouchScreener ${ }^{\mathrm{TM}}$. Of the 18 questions, 
participants answered baseline items, orienting items, and target questions. Two target questions, items 12 and 16, asked participants specifically about the alcohol and drug urine sample provided and whether the participant believed he or she would test positive for alcohol or drugs.

\subsubsection{Eight Panel Urine Alcohol and Drug Test}

Participants provided a visually monitored urine sample and a chain-of-custody process was completed to ensure that the sample provided was accurately processed. Following collection, the samples were shipped to a laboratory that utilized industry-standard enzyme assay (EA) and enzyme-immunoassay (EIA) alcohol and drug urine testing methods. Certified laboratory scientists assessed each sample for the presence of the following substances utilizing established cutoffs: alcohol, amphetamines, barbiturates, benzodiazepines, cannabis, cocaine, opiates, and phencyclidine (PCP). The end result was either "detected" or "not detected" for each of the aforementioned eight substances To assess the validity of each sample, a dilution and adulteration assessment occurred, suggesting whether or not the sample provided was both valid and interpretable. Samples that were found to be diluted or adulterated were considered indicative of a positive test result, which was consistent with how many court-ordered drug and alcohol treatment programs in coastal Southern California handle diluted or adulterated samples.

\section{Results}

A total of 101 volunteer participants were recruited for the study, each of whom signed an informed consent and received ten dollars $(\$ 10.00)$ for their participation. Table 1 presents a breakdown of demographic data related to the participants in this study, with a majority self-identifying as Caucasian, male, and single. 101 participants were considered robust enough to produce a medium effect size (Cohen, 1992).

Table 1. Demographic Information for Study Participants $(N=101)$.

\begin{tabular}{lll}
\hline Demographic Variables & Frequency & Percentage \\
\hline Gender & 95 & $94 \%$ \\
Male & 6 & $6 \%$ \\
Female & & \\
Ethnicity & 52 & $51 \%$ \\
Caucasian/White & 13 & $13 \%$ \\
African-American/Black & 32 & $32 \%$ \\
Latino/a & 4 & $4 \%$ \\
Other & & \\
Relationship Status & 82 & $81 \%$ \\
Single & 7 & $7 \%$ \\
Divorced & 10 & $10 \%$ \\
Married & 2 & $2 \%$ \\
Separated & 0 & $0 \%$ \\
\hline Widowed & & \\
\hline
\end{tabular}

Table 2 presents the results from the standard, eight-panel urinalysis conducted on each participant's voluntary urine sample. Ten (10) samples tested positive for one or more illicit or prohibited substance, and three (3) samples produced a diluted result, a total of $13 \%$ of the overall participant sample. Eighty-eight (88) participant samples tested negative for any illicit or prohibited substance, a total of $87 \%$ of the overall sample.

Table 2. Alcohol and Drug Urine Test Results for Study Participants $(N=$ 101).

\begin{tabular}{lll}
\hline Alcohol and Drug Urine Test Results & Frequency & Percentage \\
\hline Alcohol & 0 & $0 \%$ \\
Amphetamine & 0 & $0 \%$ \\
Benzodiazepine & 1 & $1 \%$ \\
Cocaine & 1 & $1 \%$ \\
Marijuana / THC & 3 & $3 \%$ \\
Methamphetamine & 5 & $5 \%$ \\
Dilute & 3 & $3 \%$ \\
Negative Urinalysis Results & 88 & $87 \%$ \\
\hline
\end{tabular}

Table 3 presents the results of the Veracity TouchScreener $^{\mathrm{TM}}$ and the classification of participants based on their cumulative responses to the 18 TouchScreener $^{\mathrm{TM}}$ questions. Participants were classified into one of the three following categories: (1) suspected deception based on multiple, significant psychophysiological responses (SPRs) to targeted items; (2) affirmative admission of substance use via the TouchScreener ${ }^{\mathrm{TM}}$ questions; or (3) negative (non-deceptive).

Table 3. Veracity Results for Study Participants $(N=101)$.

\begin{tabular}{lll}
\hline Veracity Results & Frequency & Percentage \\
\hline SPR Positive & 8 & $7.9 \%$ \\
Admission to Use & 13 & $12.9 \%$ \\
Negative & 80 & $79.2 \%$ \\
\hline
\end{tabular}

To assess the consistency of participant classification between the Veracity TouchScreener ${ }^{\mathrm{TM}}$ and the external criterion of the alcohol and drug urine test, Cohen's kappa was utilized. Table 4 presents the value of Cohen's kappa, which demonstrates the level of agreement, or inter-rater reliability, between the urine analysis results and TouchScreener ${ }^{\mathrm{TM}}$, as well as the classification accuracy between both measures. The results of the kappa analysis demonstrated substantial agreement between the two measures, $\mathrm{k}=.760,95 \% \mathrm{CI}$ $(.601, .918)$. With regards to the overall classification accuracy, the TouchScreener ${ }^{\mathrm{TM}}$ and alcohol and drug urine test were observed to have a high degree of agreement. Further analysis demonstrated a high degree of specificity and negative predictive value, with a more modest degree of sensitivity and positive predictive value. It should be noted that participants who admitted to using drugs or alcohol on the Veracity Touchscreener ${ }^{\mathrm{TM}}$ were classified as true positives, even if their urine analysis results were negative. A total of eight (8) participants satisfied this criterion. How the algorithm correction impacted the results of the present analysis will be examined further in the discussion section. 
Table 4. Classification Table Study Participants $(N=101)$.

\begin{tabular}{llll}
\hline & & UA Results & \\
\cline { 3 - 4 } & & Positive & Negative \\
\hline \multirow{2}{*}{ Veracity Results } & Positive & 17 & 4 \\
& Negative & 4 & 76 \\
\hline
\end{tabular}

Table 5. Classification Accuracy Table

\begin{tabular}{ll}
\hline Kappa & $\mathbf{7 6 0}$ \\
\hline Sensitivity & $81.0 \%$ \\
Specificity & $95.0 \%$ \\
Accuracy & $92.1 \%$ \\
Positive Predictive Value & $81.0 \%$ \\
Negative Predictive Value & $95.0 \%$ \\
\hline
\end{tabular}

\section{Discussion}

The current study examined the utility and viability of the Veracity TouchScreener ${ }^{\mathrm{TM}}$ as a means of detecting participants alcohol and drug use. In order to measure the classification accuracy of the TouchScreener ${ }^{\mathrm{TM}}$, an alcohol and drug urine test was employed as an external criterion. This decision was based on urinalysis being the most commonly used measure for detecting alcohol and drug use, with confirmatory analysis being the current gold standard (Lum \& Mushlin, 2004; NIDA, 1986; Verstraete, 2004). The results of the present study demonstrated that there was substantial agreement between these two measures, with an overall agreement of $92.1 \%$. This was consistent with results found in previous research (Pizitz et al., 2014). The results of the current study also demonstrated that the TouchScreener ${ }^{\mathrm{TM}}$ had a high degree of specificity and negative predictive value with a more modest degree of sensitivity and positive predictive value. More specifically, with respect to the current study, the TouchScreener $^{\mathrm{TM}}$ held a $95 \%$ probability of accurately identifying negative results. Conversely, it demonstrated a $5 \%$ probability of providing a false negative result. This was exemplified by the four (4) participants who were falsely identified as being negative by the TouchScreener ${ }^{\mathrm{TM}}$, but actually provided a positive urinalysis result.

The results of the present study further demonstrate how self-admission appears to be one of the key processes that contribute to the effectiveness and accuracy of the TouchScreener ${ }^{\mathrm{TM}}$. This appears to be supported as thirteen (13) of the twenty-one (21) positive test results on the TouchScreener ${ }^{\mathrm{TM}}$ were attributable to self-admission. In comparison to urinalysis, self-admissions alone would have yielded an equal number of positive results. Moreover, eight (8) participants who admitted to using substances on the TouchScreener ${ }^{\mathrm{TM}}$ provided a negative urine test.

Those participants who admitted to using alcohol or drugs on the touch screen, but provided a negative urine test, were classified as true positives. This resulted in eight (8) participants being reclassified as "true positives" rather than "false positives." Consequently, the total number of positives results for urine analysis artificially increased from thirteen (13) to twenty-one (21). In other words, the urinalysis test did not detect substance use when it had occurred, but when asked about drug use via the touch screen, these participants admitted to use.

The results of the present study further demonstrate how self-admission appears to be one of the key processes that contribute to the effectiveness and accuracy of the obtaining and maintaining sobriety. In clinical practice, having additional proven and validated tools to treat and test alcohol and drug users are of benefit. Although promising, this technology is not without limitations.

While this TouchScreener ${ }^{\mathrm{TM}}$ in this current and previous study demonstrated utility in detecting alcohol and drug use, it appears unlikely that it will replace urinalysis. This technology is unable to provide an objective measurement of the amount of a drug or corresponding metabolites that were in the participants system at the time of testing. The laboratory confirmation process is the gold standard because it possesses this ability. Nevertheless, this technology, with more rigorous testing, may still emerge as an alternative or adjunct to urinalysis as a screening measure. Based on the results of the current study, only $7.9 \%$ of participants in this study would have required follow up urine testing because of how they answered questions on the touch screen.

Other limitations of this research include the sample consisted of $94 \%$ males, with $81 \%$ of the overall participants being single and $51 \%$ being Caucasian. The sample was obtained from the San Diego metropolitan area and did not include a variety of locations. As such, the results of the current study may not be generalizable to other populations and geographical regions. The predominately male sample was moderately representative of court-ordered populations that are mostly of the male gender. According to The United States Department of Justice. (2011) Federal Justice Statistics, an estimated $75 \%$ of males and $25 \%$ of females were under Federal Probation Supervision for drug-related convictions in the year 2011. Future research should include samples with more females to determine if gender differences exist.

Future studies should attempt to replicate and validate the results of the current study by examining the classification accuracy, and most specifically the sensitivity, of this technology in relation to alcohol and urine drug tests. This will provide more evidence as to whether or not the touch screen indeed has a greater degree of sensitivity to alcohol and drug use as compared to urinalysis. Future studies should also examine the degree of agreement between this technology and other alcohol and drug detection measures such as hair follicle testing, oral swabs, and blood, as well as other psychophysiological assessment measures (i.e., polygraph or voice stress analyzer). Additionally, future studies should choose to examine this technology with various demographic and geographical samples in order to replicate the results of the current study and improve the generalizability of results.

\section{Declaration of Conflicting Interests}

The primary author is the Clinical Director for Veracity Security Solutions, LLC and one of the corresponding authors is a Business Development Analyst for Veracity Security Solutions. 


\section{Funding Sources}

The current research was funded exclusively by Veracity Security Solutions, LLC.

\section{Appendix}

A - Veracity TouchScreener ${ }^{\mathrm{TM}}$ Questions

1. Are you a citizen of this country?

2. Are you a living with someone 17 years of age or younger?

3. Are you a living with another adult?

4. Do you understand that you must answer every question concerning alcohol and drugs truthfully?

\section{(Control/Relevant Issue)}

5. Do you know the current day of the week?

6. Do you intend to neglect or violate any part of your treatment program?

7. Do you intend to try and fake or alter your alcohol or drug test?

8. Have you attempted to obtain any information on how to fake or alter an alcohol or drug test?

(Alcohol Relevant Issue)

9. Do you know the current month?

10. Have you consumed any alcohol in the past month?

11. Have you consumed any alcohol in the past month that you have not discussed with your counselor?

12. Do you believe that the sample that you are providing today will be positive for alcohol?

(Drugs Relevant Issue)

13. Do you know the current year?

14. Have you ingested or consumed any drugs in the past month?

15. Have you ingested or consumed any drugs in the past month that you have not discussed with your counselor?

16. Do you believe that the sample you are providing today will be positive for illegal drugs?

17. Do you know what the current day of the week it is?

18. Have you taken this touch screen test for the use of alcohol and illegal drugs before?

\section{References}

[1] American Psychiatric Association. (2000). Diagnostic and statistical manual of mental disorders - text revision
(DSM-IV-TR). (4th ed.). Washington, DC: Author.

[2] Cohen, J. (1992). Statistical power analysis. Current Directions In Psychological Science, 1(3), 98-101

[3] Kelly, K., Murray, E., Barrios, V., Gorman, J., Ganis, G., \& Keenan, P. (2009). The effect of deception on motor cortex excitability. Social Neuroscience, 4 (6), 570- 574

[4] Lum, G., \& Mushlin, B. (2004). Urine drug testing: Approaches to screening and confirmation testing. Laboratory Medicine, 35(6), 368-373.

[5] MacDonald, I. (April 22, 2013). The GOP's drug-testing dragnet. How republicans and industry profiteers are targeting high school students, welfare applicants and the unemployed. The Nation. Retrieved from http://www.thenation.com/article/173654/gops-drug-testing-dr agnet

[6] Melanson, S. E., Baskin, L., Magnani, B., Kwong, T. C., Dizon, A., \& Wu, A. H. (2010). Interpretation and utility of drug abuse immunoassays: lessons from laboratory drug testing surveys. Archives of Pathology \& Laboratory Medicine, 134(5), 735-739. doi: 10.1043/1543-2165-134.5.735.

[7] National Institute on Drug Abuse. (1986). Research Monograph Series 73. Urine Testing for Drugs of Abuse. R. L. Hawks, \& C. N. Chiang (Eds.). Rockville, MD: National Institute on Drug Abuse.

[8] Office of the Federal Register. (2011). Code of Federal Regulations, Title 10, Energy, Pt. 1-50, Revised as of January 1, 2011. Washington, DC: National Archives and Records Administration, Office of the Federal Register.

[9] Pizitz, T. D., McCullaugh, J. M., Sprague, P., Vaccaro, A., Blue, J., Mealing, D., \& Fernandez, K. (2014). Measuring the veracity of alcohol and drug users' self-report of sobriety. International Journal of Clinical Psychiatry and Mental Health, 2, 70-76.

[10] United States Department of Justice. (2011). Federal justice statistics, 2011 - statistical tables. Retrieved from http://www.bjs.gov/content/pub/pdf/fjs11st.pdf

[11] Venkatratnam, A., \& Lents, N. (2011). Zinc Reduces the Detection of Cocaine, Methamphetamine and THC by ELISA Urine Drug Testing. Journal of Analytical Toxicology, 35, July/August 2011.

[12] Verstraete, A. G. (2004). Detection Times of Drugs of Abuse in Blood, Urine, and Oral Fluid. Therapeutic Drug Monitoring, 26, 200-205. 